\title{
Crystal Structure of 7-Methoxy-4-[(E)-oct-1-enyl]pyrrolo[1,2-a]quinoxaline
}

\author{
Jean Guillon, ${ }^{\dagger \dagger}$ Isabelle Forfar, * Vanessa DeSPlat, $* *$ Solène Belisle FABRe,* \\ Stéphane MASSIP, ${ }^{*}$ Djavad MossalaYI, ${ }^{* *}$ and Christian JARRY* \\ *EA 4138-Pharmacochimie, UFR des Sciences Pharmaceutiques, Université Bordeaux 2, 146 rue \\ Léo Saignat, 33076 Bordeaux Cedex, France \\ **PPF Médicaments-Parasitologie, UFR des Sciences Pharmaceutiques, Université Bordeaux 2, 146 rue \\ Léo Saignat, 33076 Bordeaux Cedex, France
}

\begin{abstract}
The X-ray crystal structure of the antileishmanial 7-methoxy-4-[(E)-oct-1-enyl]pyrrolo[1,2-a]quinoxaline was established. It crystallizes in the monoclinic space group $P 2{ }_{1} / c$. Solid-state data could be used to clarify the mechanism of action implicating this new antileishmanial 4-(E)-alkenylpyrrolo[1,2-a]quinoxaline.
\end{abstract}

\section{(Received November 9, 2007; Accepted December 26, 2007; Published on wed February 26, 2008)}

Leishmaniasis is caused by different species belonging to the genus Leishmania, a protozoan that is transmitted to humans by the bite of an insect vector, phlebotomine sandfly. Leishmaniasis has an overwhelming impact on global public health, and is endemic in many tropical and subtropical regions of the world. It affects around 12 million people of the world, and 350 million are estimated to be prone to the disease, of which around 1.7 million people will be infected each year. The infection by various strains of Leishmania causes a wide spectrum of disease in humans, with many different clinical manifestations, that is, cutaneous, mucocutaneous and visceral. ${ }^{1}$

In the course of our work on the synthesis of new

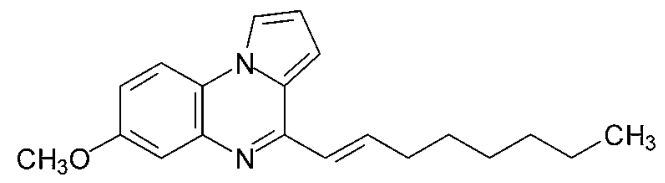

Fig. 1 Chemical structure of 7-methoxy-4-[(E)-oct-1enyl]pyrrolo[1,2-a]quinoxaline.

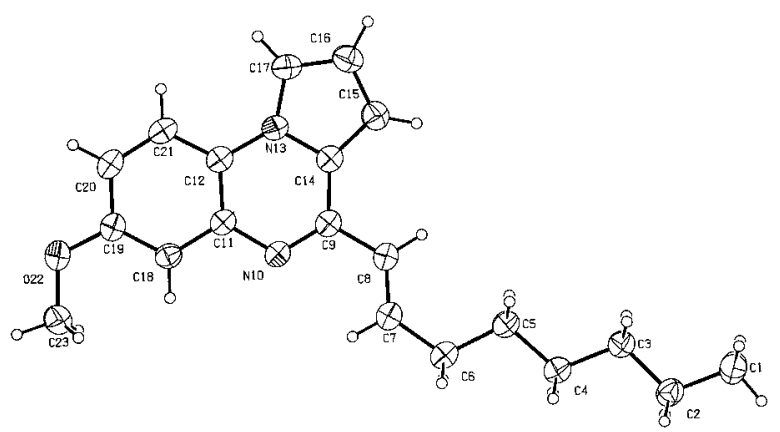

Fig. 2 View of the crystal structure of 7-methoxy-4-[(E)-oct-1enyl]pyrrolo[1,2-a]quinoxaline with our numbering scheme. Displacement ellipsoids are drawn at the $30 \%$ probability level.

$\dagger$ To whom correspondence should be addressed. E-mail: Jean.Guillon@chimphys.u-bordeaux2.fr antileishmanial drugs, we have focused our interest in the synthesis of 4-(E)-alkenylpyrrolo[1,2- $a]$ quinoxalines, ${ }^{2,3}$ new structural analogues of alkaloid chimanine B. ${ }^{4}$ These 4- $(E)$ alkenylpyrrolo[1,2-a]quinoxalines were synthesized in good yields using efficient palladium(0)-catalyzed Suzuki-Miyaura cross-coupling reactions. ${ }^{3}$

\section{Table 1 Crystal and experimental data}

Formula: $\mathrm{C}_{20} \mathrm{H}_{24} \mathrm{~N}_{2} \mathrm{O}$

Formula weight: 308.41

Crystal system: monoclinic $\quad Z=4$

Space group: $P 2_{1} / c$

$a=7.803(10) \AA$

$b=18.905(10) \AA$

$c=11.906(2) \AA$

$\alpha=90^{\circ}$

$\beta=104.10(10)^{\circ}$

$\gamma=90^{\circ}$

$V=1703.4(4) \AA^{3}$

Crystal size $=0.10 \times 0.10 \times 0.25 \mathrm{~mm}^{3}$

$D_{\mathrm{x}}=1.203 \mathrm{Mg} / \mathrm{m}^{3}$

$F\left(\begin{array}{lll}0 & 0 & 0\end{array}\right)=664$

$\mu=0.610 \mathrm{~mm}^{-1}$

Temperature $=293(2) \mathrm{K}$

$\theta_{\max }=64.92^{\circ}$

Radiation: $\mathrm{Cu} K_{\alpha}(\lambda=1.54180 \AA)$

No. of reflections measured $=2781$

No. of reflections used $=1913$ with $I>2 \sigma(I)$

$R 1=0.0479$

$w R=0.1148$

Goodness-of-fit $=1.061$

$(\Delta / \sigma)_{\max }=0.002$

$(\Delta \rho)_{\max }=0.186 \AA^{-3}$

$(\Delta \rho)_{\min }=-0.178 \AA^{-3}$

Measurement: Enraf-Nonius CAD4

Structure determination: SHELXS-976

Refinement: SHELXL-977

Crystallographic Data Centre as supplementary publication number CCDC-626085. Cambridge Crystallographic Data Centre, University Chemical Lab, 12 Union Road, Cambridge, CB2 1EZ, UK. 
Table 2 Final atomic coordinates and equivalent isotropic thermal parameters $\left(U_{\mathrm{eq}}\left(\AA^{2}\right)\right)$

\begin{tabular}{lcccc}
\hline Atom & $x$ & $y$ & $z$ & $U_{\text {eq }}$ \\
\hline $\mathrm{C}(1)$ & $0.2028(3)$ & $0.1364(1)$ & $-0.2072(2)$ & $0.0081(1)$ \\
$\mathrm{C}(2)$ & $0.1493(3)$ & $0.2113(1)$ & $-0.2446(2)$ & $0.0066(1)$ \\
$\mathrm{C}(3)$ & $0.2028(3)$ & $0.2655(1)$ & $-0.1491(2)$ & $0.0058(1)$ \\
$\mathrm{C}(4)$ & $0.1455(3)$ & $0.3399(1)$ & $-0.1875(2)$ & $0.0057(1)$ \\
$\mathrm{C}(5)$ & $0.1945(3)$ & $0.3972(1)$ & $-0.0963(2)$ & $0.0057(1)$ \\
$\mathrm{C}(6)$ & $0.1311(3)$ & $0.4693(1)$ & $-0.1451(2)$ & $0.0061(1)$ \\
$\mathrm{C}(7)$ & $0.1746(3)$ & $0.5318(1)$ & $-0.0669(2)$ & $0.0058(1)$ \\
$\mathrm{C}(8)$ & $0.2760(3)$ & $0.5348(1)$ & $0.0382(2)$ & $0.0058(1)$ \\
$\mathrm{C}(9)$ & $0.3098(2)$ & $0.6004(1)$ & $0.1064(2)$ & $0.0054(1)$ \\
$\mathrm{N}(10)$ & $0.2536(2)$ & $0.6602(1)$ & $0.0553(1)$ & $0.0055(1)$ \\
$\mathrm{C}(11)$ & $0.2835(2)$ & $0.7220(1)$ & $0.1212(2)$ & $0.0050(1)$ \\
$\mathrm{C}(12)$ & $0.3713(2)$ & $0.7236(1)$ & $0.2386(2)$ & $0.0051(1)$ \\
$\mathrm{N}(13)$ & $0.4336(2)$ & $0.6588(1)$ & $0.2891(2)$ & $0.0052(1)$ \\
$\mathrm{C}(14)$ & $0.4034(2)$ & $0.5965(1)$ & $0.2253(1)$ & $0.0054(1)$ \\
$\mathrm{C}(15)$ & $0.4801(3)$ & $0.5424(1)$ & $0.2987(2)$ & $0.0066(1)$ \\
$\mathrm{C}(16)$ & $0.5575(3)$ & $0.5724(1)$ & $0.4061(2)$ & $0.0070(1)$ \\
$\mathrm{C}(17)$ & $0.5280(3)$ & $0.6434(1)$ & $0.3988(2)$ & $0.0063(1)$ \\
$\mathrm{C}(18)$ & $0.2215(3)$ & $0.7858(1)$ & $0.0655(1)$ & $0.0055(1)$ \\
$\mathrm{C}(19)$ & $0.2467(3)$ & $0.8486(1)$ & $0.1257(2)$ & $0.0057(1)$ \\
$\mathrm{C}(20)$ & $0.3312(3)$ & $0.8486(1)$ & $0.2434(2)$ & $0.0062(1)$ \\
$\mathrm{C}(21)$ & $0.3924(3)$ & $0.7871(1)$ & $0.2992(2)$ & $0.0059(1)$ \\
$\mathrm{O}(22)$ & $0.1925(2)$ & $0.9135(1)$ & $0.0795(2)$ & $0.0074(1)$ \\
$\mathrm{C}(23)$ & $0.1035(3)$ & $0.9158(1)$ & $-0.0398(2)$ & $0.0078(1)$ \\
\hline
\end{tabular}

$U_{\text {eq }}=(1 / 3) \Sigma_{i} \Sigma_{j} U_{i j}\left(a_{i} * a_{j}^{*}\right)\left(\boldsymbol{a}_{i} \cdot \boldsymbol{a}_{j}\right)$.

We report herein the structural characterization of one of them, i.e. the 7-methoxy-4-[(E)-oct-1-enyl]pyrrolo[1,2a]quinoxaline (Fig. 1), which shows antileishmanial activity in vitro upon the Leishmania amazonensis $\left(\mathrm{IC}_{50}=2 \mu \mathrm{M}\right)$ and Leishmania infantum strains $\left(\mathrm{IC}_{50}=1.2 \mu \mathrm{M}\right)$.

Pale-yellow crystals suitable for X-ray analysis were obtained by slow crystallization from an ethanol solution at $0^{\circ} \mathrm{C}$. The structure of 7-methoxy-4-[(E)-oct-1-enyl]pyrrolo[1,2a]quinoxaline is illustred in Fig. 2. Crystal and experimental data are given in Table 1.

The 3D spatial structure of the 4-octenylpyrrolo[1,2a]quinoxaline showed the $(E)$-isomerism of the 4-lateral $\mathrm{C}_{8} \alpha$ unsaturated alkenyl chain in the solid state, as anticipated based on ${ }^{1} \mathrm{H}$ NMR data. ${ }^{3}$ The trans $\mathrm{C}(8)-\mathrm{C}(7)$ octenyl double bond was noticed at $1.308(3) \AA$, as typically observed for $\mathrm{C}=\mathrm{C}$ double bonds.

The pyrrolo[1,2-a]quinoxaline moiety is almost planar, and the maximum deviation from planarity is found for $\mathrm{C}(21)$ lying $-0.0414(2) \AA$ from the plane defined by the hetero-tricyclic
Table 3 Hydrogen-bonding geometry $\left(\AA,^{\circ}\right)$

\begin{tabular}{cccl}
\hline$C$-H...A & H-A & $C$-A & $N$-H...A \\
\hline $\mathrm{C}(7)-\mathrm{H}(7) \ldots \mathrm{N}(10)$ & 2.46 & $2.820(3)$ & 103 \\
\hline
\end{tabular}

system. The 4-(E)-octenyl chain was also found to be quite planar; a deviation of the $C(5)$ atom was noticed at $-0.0586(2) \AA$ from the least-square plane defined by the other atoms of the alkenyl group. The angle between the least-squares planes, defined by the pyrroloquinoxaline moiety and the alkenyl chain, was approximatively $4.18(1)^{\circ}$. The final atomic coordinates and the equivalent isotropic thermal parameters are listed in Table 2.

The alkenyl chain substituted at position 4 adopts a quite regular conformation, described by the torsion angles $\mathrm{C}(1)-\mathrm{C}(2)-\mathrm{C}(3)-\mathrm{C}(4), \quad \mathrm{C}(2)-\mathrm{C}(3)-\mathrm{C}(4)-\mathrm{C}(5)$, $\mathrm{C}(3)-\mathrm{C}(4)-\mathrm{C}(5)-\mathrm{C}(6), \quad \mathrm{C}(4)-\mathrm{C}(5)-\mathrm{C}(6)-\mathrm{C}(7) \quad$ and $\mathrm{C}(6)-\mathrm{C}(7)-\mathrm{C}(8)-\mathrm{C}(9)$, which are $-179.0(2), 180.0(2), 179.7(2)$, $-177.9(2)$ and $179.4(2)^{\circ}$, respectively. However, the $\mathrm{C}(5)-\mathrm{C}(6)-\mathrm{C}(7)-\mathrm{C}(8)$ angle was noticed at $6.9(3)^{\circ}$, involving such a conformation of this 4-lateral chain through rotation around the $\mathrm{C}(6)-\mathrm{C}(7)$ bond.

There are no solvent accessible voids in the crystal lattice of the 4-(E)-octenylpyrrolo[1,2- $a]$ quinoxaline. The structure exhibits an intramolecular hydrogen bond of the type $\mathrm{C}-\mathrm{H} \cdots \mathrm{N}$ (Table 3).

\section{References}

1. S. L. Croft and V. Yardley, Curr. Pharm. Des., 2002, 8, 319.

2. J. Guillon, I. Forfar, M. Mamani-Matsuda, V. Desplat, M. Saliège, D. Thiolat, S. Massip, A. Tabourier, J.-M. Léger, B. Dufaure, G. Haumont, C. Jarry, and D. Mossalayi, Bioorg. Med. Chem., 2007, 15, 194.

3. J. Guillon, I. Forfar, V. Desplat, S. Belisle Fabre, D. Thiolat, S. Massip, H. Carrie, D. Mossalayi, and C. Jarry. J. Enz. Inhib. Med. Chem., 2007, 22, 541.

4. A. Fournet, R. Hocquemiller, F. Roblot, A. Cavé, P. Richomme, and J. Bruneton, J. Nat. Prod., 1993, 56, 1547.

5. Enraf-Nonius CAD-4 Software, Version 5.0, 1989, EnrafNonius: Delft, The Netherlands.

6. G. M. Sheldrick, SHELXS 97, Program Crystal Structure Solution, 1997, University of Göttingen, Germany.

7. G. M. Sheldrick, SHELXL 97, Program for Crystal Structure Refinement, 1997, University of Göttingen, Germany. 